\title{
Inferring decay in short-term memory: The issue of capacity
}

\author{
HENRY L. ROEDIGER, III, JAMES L. KNIGHT, JR., and BARRY H. KANTOWITZ \\ Purdue University, West Lafayette, Indiana 47907
}

\begin{abstract}
Experiments examining the issue of decay in short-term memory have assumed a single undifferentiated source of processing capacity which cannot be devoted to rehearsal when consumed in the processing of a nonverbal interpolated task. Three experiments reported here call this logic into question, since variations in difficulty in the nonverbal interpolated task failed to affect recall. Slight forgetting produced by a nonverbal interpolated task, relative to a no interpolated task control, was attributed to qualitative differences from performing two tasks simultaneously rather than only one. Results from the third experiment indicated that retrieval after a period of nonverbal interpolated activity is from primary rather than secondary memory.
\end{abstract}

The idea that forgetting is at least partly attributable to autonomous decay of the memory trace has suffered several reversals of fortune during this century. The most prevalent theory of forgetting early in the century was that "the machinery developed in the process of learning is subject to the wasting effects of time" (Woodworth, 1929, p. 93). McGeoch (1932) mounted so effective an attack on decay theory that evidently no one seriously considered it as an appropriate account of forgetting for 25 years. Brown (1958) resurrected decay theory to account for forgetting over short retention intervals for small amounts of material when subjects were distracted during the interval by performing another task, such as number shadowing. Decay was plausible, since Brown's (1958) experiments seemed to show that proactive and retroactive inhibition were relatively unimportant in this situation. However, later research has clearly demonstrated that proactive and retroactive inhibition are powerful factors determining forgetting in the Brown-Peterson distractor task (Brown, 1958; Peterson \& Peterson, 1959). In fact, on the first trial (i.e., without proactive interference) in experiments with this technique, there is little, if any, forgetting (Keppel \& Underwood, 1962).

Recently the issue of decay in immediate memory has been reopened in an important series of studies by Reitman (1971, 1974), Shiffrin (1973), and Watkins, Watkins, Craik, and Mazuryk (1974). The purpose of the present paper is to consider critically the logic used in these investigations to draw inferences about decay. These investigations capitalize on the similarity

Order of authors was randomly determined. The present research was supported by NIMH Grant MH26302 to Barry $\mathrm{H}$. Kantowitz. We thank Fred Hoehler for testing subjects in Experiment 3 and Robert G. Crowder for commenting on the manuscript. Requests for reprints should be sent to any author at the Department of Psychology, Purdue University, West Lafayette, Indiana 47907. of the Brown-Peterson short-term memory task to procedures used in the study of divided attention, a similarity first noted by Crowder $(1967 a, b)$. During the retention interval in short-term memory experiments, there is competition between tendencies to rehearse the memory stimuli and to execute an interpolated "filler" activity. This characteristic of the Brown-Peterson paradigm has been exploited in two related but not entirely complimentary ways. First, by varying the processing capacity demands of the interpolated task, experimenters have attempted to control rehearsal. Thus, effects of the interpolated task upon memory were at issue. Second, attempts have been made to gain an index of covert rehearsal by comparing interpolated task performance in the presence of memory load to performance of the interpolated task in isolation. Here interest focused on effects of the memory task upon the interpolated task.

Reitman (1971) attempted to apply this methodology to the study of decay in immediate memory by selecting an interpolated task that would demand capacity but was quite different in nature from the verbal memory task, thus minimizing retroactive interference. With subjects' limited processing capacities engaged with the nonverbal interpolated task during the retention interval, rehearsal should be minimized and any forgetting would presumably reflect decay, rather than retroactive interference from the interpolated task. Furthermore, any tendency to rehearse covertly should be detectable by the concomitant decrement in interpolated task performance relative to a control condition where subjects performed only the interpolated task.

Reitman (1971) reported almost perfect retention of three-word lists following a $15-\mathrm{sec}$ retention interval filled with the detection of pure tones in white noise. Since the detection task was not performed better in isolation than when combined with the memory task, Reitman concluded that (1) there was no covert re- 
hearsal and (2) there was no decay in immediate memory. Shiffrin (1973) replicated Reitman's findings of no forgetting and no interpolated task decrement in three more experiments involving recall of five-letter strings following retention intervals of up to $40 \mathrm{sec}$ filled with tone detection.

The conclusion that short-term memory traces do not decay was called into question by further experiments by Watkins et al. (1974) and Reitman (1974). Watkins et al. (1974, Experiment 1) found increasingly greater forgetting of five-word lists over a $20-\mathrm{sec}$ retention interval filled with a four-choice tone discrimination task. Such forgetting was not found when subjects rehearsed or simply listened to tones during the interval. A similar finding was reported in a second experiment where subjects engaged in pursuit-rotor tracking during retention. Watkins et al. (1974) reconciled the discrepancy between their results and those of Reitman (1971) and Shiffrin (1973) by arguing that the tone detection task used by the latter investigators simply did not use all available processing capacity. In a later experiment Reitman (1974) also argued that her earlier conclusion of no decay was in error due to ceiling effects in the memory task and insufficiently sensitive methods for measuring rehearsal effects on the tone detection task.

Applications, such as those described above, of the divided-attention logic to the decay issue in short-term memory involve many pitfalls in the form of assumptions that must be verified before justifiable conclusions can be drawn (Kantowitz, 1974; Kerr, 1973). Two aspects of this issue were previously discriminated: effects of memory upon the interpolated task and effects of the interpolated task upon memory. We will now focus on just one of these aspects, the conditions under which interpolated task performance can be validly interpreted as an index of covert rehearsal.

Consider Reitman's (1971) short-term memory experiment utilizing the Brown-Peterson paradigm to examine the decay issue. In this study interpolated task performance was examined (1) in isolation (i.e., in a single-task control condition) and (2) in concert with a memory task (i.e., in a dual-task condition). A possible finding in such an experiment, and the one that was obtained by Reitman (1971), is no change in interpolated task performance between single- and dualtask conditions. Such a result is ambiguous, since it could indicate either that rehearsal has no effect upon the interpolated task or that no rehearsal occurred. Reitman drew the latter conclusion, but evidence must be offered to show that rehearsal potentially interferes with the interpolated task. The test of this assumption is crucial since recent studies have demonstrated that some tasks may not require processing capacity and therefore do not interfere with other concurrent processing (Kerr, 1973). Alternatively, rehearsal may not interfere with the interpolated task if the capacity demands of these two components are restricted to different processing stages, each with an independent supply of capacity (e.g., Brooks, 1968; Kantowitz \& Knight, 1976a).

A plausible alternative finding in Reitman's (1971) experiment would have been decreased interpolated task performance in dual- as opposed to single-task conditions. This result would also be ambiguous. It might reflect an effect of rehearsal upon the interpolated task, as assumed by Reitman (1971), but on the other hand, such an interpolated task performance decrement might be due to factors unrelated to the capacity demands of rehearsal but associated with qualitative differences between single- and dual-task conditions (Kalsbeek \& Sykes, 1970; Kantowitz \& Knight, 1974). Such nonspecific, qualitative differences may involve a variety of possible changes. For example, such differences may reflect changes in arousal or effort (Kahneman, 1974) when subjects are faced with performing two tasks rather than only one. Furthermore, the criteria against which subjects calibrate their performance may vary between single- and dual-task conditions (Kantowitz, 1974; Kantowitz \& Knight, 1976b). Another possibility includes gross alterations in strategies governing performance, such as shifting from an "error correction" mode in tracking to a "pattern generation" mode (Pew, 1974). Structural interference may be appreciable if the tasks being combined require incompatible responses or compete for a single sensory system, as when both tasks are visually presented. Yet another source of qualitative difference may lie in task timing parameters. For example, recall may be more delayed in dual- relative to single-task conditions if in dual-task conditions subjects attempt either to complete some aspect of an interpolated task prior to initiating recall or to link dual tasks according to some temporal coding constraint.

In general, it is not possible to discriminate performance changes (in either the memory or interpolated tasks) due to nonspecific qualitative factors from changes due to reallocation of limited processing capacities when single-task performance is contrasted with performance in only one dual-task condition. The only compelling way to implicate capacity rather than structural limitations is to demonstrate that variations in processing capacity demands of one task produce performance changes in the other task.

All studies on the decay issue (Reitman, 1971, 1974; Shiffrin, 1973; Watkins et al., 1974) have employed only one dual-task condition. It is essential to amend the methodology of these studies so that the outcome of such experiments can be interpreted unambiguously. There are two possibilities, which we discuss in turn: (1) variation in capacity demands of the memory task and (2) variation in capacity demands of the interpolated task.

\section{Varying Memory Demands}

By varying the difficulty of the memory task to produce different dual-task conditions, a contrast can be drawn between performance on the interpolated task 
when the memory task requires either lesser or greater capacity. A potentially unambiguous result may be obtained with this methodology. If interpolated task performance differs as a function of the difficulty of the memory task, one may then reasonably infer that the particular interpolated task used in this context may be regarded as a sensitive indicant of covert rehearsal. This result was obtained by Johnston, Greenberg, Fisher, and Martin (1971), who combined pursuitrotor tracking with two difficulty levels of a concurrent memory task.

It is crucial to note that, while this outcome (changes in interpolated task performance as a function of memory task difficulty) implies that memory processing demands can limit interpolated task performance, this implication cannot be generalized without further evidence to any interpolated task. Quite conceivably, a different interpolated task might not require capacity at all or the capacity it requires might be localized in a stage with a capacity supply independent of that used for rehearsal. Thus, for example, it would be premature to conclude on the basis of the results of Johnston et al. (1971) that covert rehearsal affects interpolated task performance in studies such as those of Reitman (1971, 1974) and Shiffrin (1973), where a tone detection task was used as an interpolated task. Indeed, available evidence from studies in both vision and audition indicates that signal detection may not require capacity (e.g., Shiffrin \& Gardner, 1972; Sorkin \& Pohlmann, 1973).

In order to utilize interpolated task performance as an index of covert rehearsal, the following three assumptions must be validated: (1) Rehearsal must be shown to demand processing capacity, (2) the interpolated task must be shown to demand processing capacity, and (3) both rehearsal and the interpolated task must be shown to draw on the same source of capacity. Let us consider what conclusions can be drawn when difficulty of the memory task is varied and there is no effect on interpolated task performance. Although Reitman (1971) and Shiffrin (1973) compared only single- and dual-task conditions, they found no effect of the presence or absence of a memory load on interpolated task performance. Such a null result could reflect at least three possibilities. First, there may have been no covert rehearsal in the memory condition to interfere with the interpolated task. The interpolated task then successfully prevented rehearsal. This is the conclusion Reitman (1971) and Shiffrin (1973) drew. But two other conclusions are just as valid on the basis of only this evidence. If any or all of the three assumptions discussed above are false, a second possibility is that rehearsal and the interpolated task may proceed in parallel without interference. The third interpretation of this lack of effect of varying the memory task on interpolated task performance is that it is due to either of two special characteristics of the memory or interpolated tasks: The tasks may have been too easy, or performance may have been observed at a plateau on the performanceresource function. In this context the tasks would be "too easy" if the total processing demands of the memory and interpolated tasks together did not exceed available capacity resources. If available resources are never exceeded, no decrement on the interpolated task would be expected even with increased rehearsal demands. The second possibility is suggested by Norman and Bobrow's (1975) notion of performance-resource functions that depict task performance as a function of resources (in this case the resource of interest is processing capacity) allocated to a task. Norman and Bobrow (1975) indicated the possibility of plateaus in resource-performance functions where changes in resource allocation produce no performance changes. The failure to find an effect of increasing memory task difficulty on interpolated task performance may therefore indicate that interpolated task performance was observed at such a plateau and variations in the memory task were too small to move the interpolated task off its resource-performance plateau.

How can the ambiguity associated with this type of null result (no effect of memory load on the interpolated task) be reduced? One solution would be to vary the rehearsal requirements of the memory task over a wide range, so that the memory task might drain away enough processing capacity from the interpolated task to reveal any possible interference. The primary disadvantage of this method, and the reason we chose the alternate solution discussed below, is that it is not well suited for situations in which the interpolated task is used to eliminate as well as check for covert rehearsal (Reitman, 1971; Shiffrin, 1973). Since we are primarily concerned with the fate of memories during a nonverbal interpolated activity, the issue of capacity is better examined with variations in demands of the interpolated task.

\section{Varying Interpolated Task Capacity Demands}

The alternative approach to the problem of inferring covert rehearsal in short-term memory requires varying the processing capacity demands of the interpolated task. This solution has the advantages that (1) the possibility of observing the interpolated task at a resource-performance plateau is reduced, and (2) the same memory task can be observed in all conditions. If covert rehearsal is occurring and if the three assumptions discussed previously are valid for the interpolated task in question, then interpolated task decrements should be observed when single- and dual-task conditions are contrasted. Furthermore, if this decrement is due to capacity demands of the interpolated task rather than qualitative differences between single- and dual-task conditions, recall should be poorer with interpolated tasks requiring greater capacity. Failure to find poorer recall with increasingly difficult interpolated tasks would lead to the conclusion that one or more of the assumptions discussed previously, and on which con- 
clusions from the studies by Reitman et al. depend, is faulty.

These considerations on the issue of capacity are crucial for interpreting any potential results from experiments examining the decay issue. Despite their critical nature, their treatment in recent studies has been casual at best. (Interestingly, most of these issues were discussed in the earlier studies of Crowder, 1967a,b.) The present studies provide the essential variation in interpolated task difficulty to uncover whether or not forgetting of verbal material during a nonverbal interpolated task is due to decay when capacity is fully demanded by the interpolated task, or due simply to nonspecific or structural interference. Although Reitman (1974) and Shiffrin (1973) provide some evidence bearing on the issue of shared capacity demands of their two tasks (memory and tone detection), the most direct way of examining this question (that is, varying interpolated task difficulty) has not been utilized.

In the present experiments subjects were required to remember short lists of words during a retention interval either filled with an interpolated task (usually nonverbal) or unfilled. A tapping task (Fitts, 1954) was used as the nonverbal distractor activity. The difficulty of this task was varied in Experiments 1 and 3 by altering the physical dimensions (target width and distance between targets) of the target plates and in Experiment 2 by altering the pacing rate of reciprocal tapping between the two targets. The tapping task was chosen over tone detection for two reasons: (1) Unlike tone detection, it has been shown to require capacity when performed in concert with other tasks (Kantowitz \& Knight, 1974), and (2) it requires continuous movement rather than the discrete judgments of the delection task, so that even momentary shifts of attenticm can be monitored, if necessary.

\section{EXPERIMENT 1}

\section{Method}

Subjects. Twenty right-handed female volunteers participated to partially fulfill a course requirement.

The tapping task. The tapping task involved moving a stylus between left and right target plates in time with pacing lights which flashed from side to side at 2 flashes/sec. Tapping continued for $30 \mathrm{sec}$ on each trial involving tapping. Target plates were $7 \mathrm{~cm}$ long, and the difficulty of the tapping task was manipulated by varying the center-to-center distance between target plates (the movement amplitude $-\mathrm{A}$ ) and the width (W) of the target plates. Using movement amplitude and target width, an index of difficulty (ID, Fitts, 1954), which combines aspects of the information metric and Weber fraction, may be computed for the tapping task using the relationship $\mathrm{ID}=\log _{2} 2 \mathrm{~A} / \mathrm{W}$. For the difficult tapping condition, target widths of $1.27 \mathrm{~cm}$ and movement amplitude of $20.45 \mathrm{~cm}$ yielded an ID $=5$ bits. For the easy tapping condition, target widths were $5.08 \mathrm{~cm}$ and movement amplitude was $10.16 \mathrm{~cm}$, yielding an ID $=1.96$ bits. Since tapping was paced at 2 taps $/ \mathrm{sec}$, in the easy tapping condition subjects generated $3.91 \mathrm{bits} / \mathrm{sec}$ if tapping was errorless. In the difficult tapping condition, subjects were required to generate $10 \mathrm{bits} / \mathrm{sec}$. All target hits, overshoots, and undershoots were recorded electrically.
The memory task. In the memory task, five common words arranged in a column were simultaneously displayed by a slide projector for $5 \mathrm{sec}$, and subjects were instructed to read the words aloud. After $5 \mathrm{sec}$, the to-be-remembered words were removed and a $30-\mathrm{sec}$ retention interval began. The retention interval was filled with easy, difficult, or no tapping. A short tone signaled the end of the retention interval, and subjects were given $20 \mathrm{sec}$ to write down as many of the five stimulus words as possible. A blank computer card was provided for this purpose on each trial. Subjects were urged to take advantage of the full recall period and no instructions were given concerning order of recall. A second short tone indicated the end of the recall interval and, $5 \mathrm{sec}$ later, the next trial began.

Design and procedure. Three experimental conditions were formed by inserting easy tapping, difficult tapping, or no tapping into the 30-sec retention interval of the memory task. In two additional control conditions, subjects performed $30 \mathrm{sec}$ of easy tapping or difficult tapping without the memory task. Each subject received 10 consecutive trials in each experimental condition. The five conditions were arranged according to a 5 by 5 Latin square and four subjects were randomly assigned to each row.

Subjects were tested individually. After listening to taperecorded instructions, two memory practice trials were administered with the difficult interpolated task. The experimenter then informed the subject as to the experimental condition in the upcoming 10-trial block. The trial block then proceeded uninterrupted and successive blocks were separated by 2 -min rest periods.

\section{Results and Discussion}

Recall was scored according to a free recall criterion, that is, subjects were given credit for items correctly recalled without regard to order. Mean correct recall for the no-tapping, easy tapping, and difficult tapping conditions was $4.65,4.43$, and 4.35 words per trial, respectively. An analysis of variance performed on recall in the three conditions over the 10 trial blocks revealed a reliable effect of conditions $[F(2,38)=7.29, p<.01$, MSe $=.66]$ but no effect of trials and no interaction. While a Dunnett test showed memory performance in the no-tapping condition to be reliably different from that in the easy tapping condition $[\mathrm{d}(38)=3.82$, $\mathrm{p}<.025$ ], an even more liberal $t$ test failed to reveal any difference between recall in the easy and difficult tapping conditions $[\mathrm{t}(38)=1.39]$. Although recall was worse under conditions where a tapping task was performed during the 30-sec retention interval than when no task was performed, there was no difference between recall with an easy vs difficult tapping task.

Tapping performance was assessed by the number of on-target taps (hits). Multiplying by appropriate constants (3.91 bits/tap for easy tapping and $10 \mathrm{bits} / \mathrm{tap}$ for difficult tapping) also transformed these data to information transmitted $\left(\mathrm{H}_{\mathrm{t}}\right)$ during the 30-sec tapping interval. The mean number of hits in the easy and difficult tapping conditions is shown in Table 1 , both for trials when there was a concomitant memory task and for trials when there was no memory task. While fewer hits were made in the difficult than easy tapping condition, where the tapping plates were narrower and further apart $[F(1,19)=70.53, p<.001, \mathrm{MSe}=$ 66.52], more information was transmitted in the diffi- 
Table 1

Mean Number of Hits on the Interpolated Tapping Task as a Function of Condition in Experiment 1

\begin{tabular}{lccc}
\hline & \multicolumn{2}{c}{ Memory Condition } & \\
\cline { 2 - 3 } Tapping & $\begin{array}{c}\text { Memory } \\
\text { Load }\end{array}$ & $\begin{array}{c}\text { No Memory } \\
\text { Load }\end{array}$ & Mean \\
\hline Easy & 29.7 & 30.1 & 29.9 \\
Difficult & 27.1 & 25.8 & 26.5 \\
Mean & 28.4 & 28.0 & \\
\hline
\end{tabular}

cult tapping condition ( 265 bits) than in the easy tapping condition $(117$ bits) $[\mathrm{F}(1,19)=85.00, \mathrm{p}<.001$, $\mathrm{MSe}=102,919]$. Thus, the failure to obtain effects of tapping difficulty on recall cannot be attributed to identical tapping performance in the easy and difficult tapping conditions.

There was no reliable effect of having vs not having a memory load on number of tapping hits in Table 1, but there was a significant Memory Condition by Tapping Difficulty interaction $[\mathrm{F}(1,19)=7.92, \mathrm{p}<.05$, $\mathrm{MSe}=38.45]$. In the difficult tapping condition, adding the memory task actually produced a slight increment in tapping performance. This suggests a tradeoff of memory performance for tapping. However, even with this possible disadvantage, recall with difficult tapping did not drop reliably below that obtained for the easy tapping condition. Similarly, adding the memory task to the easy tapping condition produced a slight decrement relative to the condition with no memory task, suggesting a tradeoff favoring the memory task. Again, this tradeoff should accentuate recall differences between the easy and difficult tapping conditions, yet no difference was observed. Thus, the interaction shown in Table 1 suggests that the failure of the tapping difficulty manipulation to affect recall was not due to tradeoffs between the memory and tapping tasks.

The main conclusion to be drawn from this experiment is that the memory and tapping tasks can be combined without interference. The finding of slightly greater forgetting during the retention interval when it is filled with a tapping task than without such a task may reasonably be attributed to qualitative differences between the memory task in these two cases. (Specific possibilities are outlined in the Discussion.) The important point is that increasing the processing capacity demands of the interpolated task did not affect recall, and there was no decrement in either the easy or difficult versions of the interpolated task when single- and dual-task conditions were contrasted. This result implies that one or more of the fundamental assumptions previously discussed as underlying the inference of decay during a nonverbal interpolated task is false.

\section{EXPERIMENT 2}

Some of the conclusions drawn from the first experiment require acceptance of a null hypothesis. In order to bolster these inferences, we decided to replicate the previous experiment, with one important change: the way interpolated task difficulty was varied. Interpolated task difficulty was manipulated in Experiment 2 by varying the rate of tapping rather than the width and spacing of the target plates. This alternate way of varying interpolated task difficulty might provide a more effective manipulation, since the number of taps required of subjects was much greater in the difficult than in the easy condition. This is unlike the manipulation in Experiment 1, where there were actually more correct taps in the easy than in the hard conditions. A second change in Experiment 2 was that ordered rather than free recall was requested.

\section{Method}

The design and procedure of Experiment 2 was identical to that of Experiment 1, except in two respects. In this experiment only one set of target plates was used (width $=5.08 \mathrm{~cm}$, amplitude $=10.16 \mathrm{~cm}$ ) and the difficulty of the tapping task was manipulated by varying the tapping pace. In the slow tapping condition, tapping was paced at $1 \mathrm{tap} / \mathrm{sec}$, thus requiring subjects to generate $1.96 \mathrm{bits} / \mathrm{sec}$. In the fast tapping condition, pacing was 3 taps/sec, thus requiring generation of $5.88 \mathrm{bits} / \mathrm{sec}$. Both tapping tasks were performed by themselves and in combination with a memory task. In a fifth condition subjects performed only the memory task.

Second, in Experiment 2 subjects were instructed to recall items in the correct input order (serial recall). Five numbered answer slots were provided on the back of computer cards, and subjects were asked to write each response in the slot corresponding to its position at the time of presentation.

Ten female subjects participated to fulfill a course requirement.

\section{Results and Discussion}

The recall results closely resembled those in Experiment 1 . When scored by a free recall criterion, mean recall in the no-tapping, slow tapping, and fast tapping conditions was $4.53,4.21$, and 4.23 words per trial, respectively $[\mathrm{F}(2,18)=4.62, \mathrm{p}<.05, \mathrm{MSe}=.69]$. Mean ordered recall for the three tapping conditions was $4.05,3.64$, and 3.54 words per trial, respectively $[\mathrm{F}(2,18)=4.65, \mathrm{p}<.05, \mathrm{MSe}=1.57]$. For both free and ordered scoring, Dunnett tests revealed recall in the memory-only control condition to be reliably different from recall in either the slow or fast tapping conditions. Once again, even the more liberal $t$ test failed to indicate significant difference between recall in the slow and fast tapping conditions with recall scored either way. Thus, as in Experiment 1, recall was worse in the dual-task conditions than in the memory-only condition, but within the dual-task condition no effect of tapping speed on recall was found. In fact, free recall performance was slightly better in the fast than in the slow tapping condition.

The mean number of hits in the tapping task is shown in Table 2 for the fast and slow tapping conditions, with and without a memory load. Not surprisingly, more hits were made and more information was transmitted in the more difficult, fast-paced condition (mean 
Table 2

Mean Number of Hits on the Interpolated Tapping Task as a - Function of Condition in Experiment 2

\begin{tabular}{lccc} 
& \multicolumn{2}{c}{ Memory Condition } & \\
\cline { 2 - 3 } Tapping & $\begin{array}{c}\text { Memory } \\
\text { Load }\end{array}$ & $\begin{array}{c}\text { No Memory } \\
\text { Load }\end{array}$ & Mean \\
\hline Slow & 17.8 & 18.0 & 17.9 \\
Fast & 49.5 & 50.7 & 50.1 \\
Mean & 33.6 & 34.4 & \\
\hline
\end{tabular}

hits $=50$, mean $H_{t}=196$ bits) than in the easy, slowpaced condition (mean hits $=18, \mathrm{H}_{\mathrm{t}}=70$ bits) $[\mathrm{F}(1,9)=$ $445.08, \mathrm{p}<.001, \mathrm{MSe}=446.92]$. Once again, the presence or absence of a memory load did not affect tapping, and in this experiment there was no interaction between slow and fast tapping and presence or absence of a memory load $[\mathrm{F}(1,9)<1, \mathrm{MSe}=50.83]$. There was a main effect of trials $[F(9,81)=5.80, p<.001$, MSe $=7.09 \mathrm{~J}$ and a significant Trials by Tapping Difficulty interaction $[\mathrm{F}(9,81)=5.42, \mathrm{p}<.001, \mathrm{MSe}=$ 6.85]. Tapping performance deteriorated over trials within each 10-trial block and this effect was greater in the fast-paced, difficult tapping condition.

\section{EXPERIMENT 3}

The results of the first two experiments are quite consistent. Recall of five words following a 30-sec retention interval was worse when subjects performed a tapping task during this period than when no task was required, but variation in the difficulty of the tapping task had no effect on recall. Difficulty was varied in different ways in the two experiments, and performance on the easy vs difficult forms of the task differed radically in both experiments in terms of the amount of information transmitted. Since memory is unrelated to the capacity demands of the interpolated task, these tasks can apparently proceed jointly with no decrement.

An alternate interpretation of these results was suggested by F.I.M. Craik, which led to the third experiment. One way to rationalize the lack of an effect of interpolated task difficulty on short-term memory is to assume that recall was from long-term or secondary memory; that is, the interpolated activity, whether easy or difficult, succeeded in preventing subjects from rehearsing during the retention interval so that recall was entirely from secondary memory. The level of difficulty of the tapping task had no effect, the argument might go, since both tasks were sufficient to consume all available processing capacity. The decrement in recall with an interpolated tapping task relative to the memory-only condition could be due either to the fact that recall was from primary memory in the latter condition or that more information had been transferred to secondary memory in this condition.

Since we argue that rehearsal and a nonverbal interpolated task can proceed jointly, we must maintain that recall at the end of the interpolated task is mostly from primary memory. On this point, both Reitman's $(1971,1974)$ and Shiffrin's (1973) positions are compatible with ours. In order to ascertain the status of information retrieved after a retention interval filled with a nonverbal interpolated task, we employed a recognition memory paradigm, with response latency as the primary dependent variable. We assumed (and provided an internal check on this assumption in the experiment) that information could be retrieved from primary memory faster than from secondary memory, so that latencies could be used as a sensitive diagnostic measure to determine whether information retrieved after an interpolated task was from primary or secondary memory.

In Experiment 3 subjects were presented with threeword lists and then given a yes/no recognition test after 0,20 , or $40 \mathrm{sec}$. During the 20 - and $40-\mathrm{sec}$ retention interval, subjects were either free to rehearse or performed one of three interpolated tasks: easy tapping, difficult tapping, or counting backward by threes as fast as possible. Latencies at the $0-\mathrm{sec}$ retention interval and after 20- and 40-sec intervals with no interpolated task provided us with an estimate of the recogntion retrieval latency from primary memory. Latencies following the backward-counting task provided us with an estimate of retrieval time from secondary memory, at least at the 40 -sec retention interval. The question of interest is whether or not retrieval times following retention intervals filled with the tapping tasks more closely resemble those following an unfilled interval or those following an interval of backward counting.

\section{Method}

Subjects. Eighteen volunteers of both sexes participated as part of a course requirement in introductory psychology.

Design. There were eight delayed test conditions formed by combining two retention intervals (20 and $40 \mathrm{sec}$ ) with four types of activity which could occur during the retention interval. The four activities were easy and difficult versions of a paced tapping task; unpaced, but rapid, backward counting; and an unfilled retention interval. One additional condition involved an immediate (that is, zero delay) test of memory. Thus, there were a total of nine conditions, and these conditions were arranged in nine sequences according to a 9 by 9 Latin square, with two subjects randomly assigned to each row of the square.

Procedure. Subjects were tested individually. Before receiving the experimental conditions, subjects were given one trial of practice counting backward by threes without the memory task. Practice trials were also given on the tapping task alone for both the easy and difficult tapping conditions. Trials in the experimental conditions followed immediately after the practice trials.

In each condition subjects experienced 12 recognition test trials in a block, and at the start of each 12-trial block the subject was informed as to the requirements of the upcoming condition. A PDP-8/L computer equipped with a crystal timebased clock was used to control stimulus presentation, data recording, and temporal sequencing. Each trial began with the presentation of three words, arranged vertically, for $4.1 \mathrm{sec}$ on the screen of a Tektronix 5103N display. Next followed the appropriate delay and interpolated activity. For the eight delay 
conditions three asterisks appeared at the bottom center of the display, where the recognition test word would later appear. If the trial required counting backward by threes, a random number between 501 and 699 appeared at the top of the display as soon as the three study words were removed. Subjects began counting aloud as rapidly as possible from this starting point. In all delay conditions the pacing lights of the tapping apparatus began to flash from side to side, as in Experiment 1 , as soon as the study words were removed. The flashing lights were present even if the interpolated activity did not involve tapping (the unfilled and backward-counting conditions) to equate for any possible visual distraction effects. If the interpolated activity involved tapping, the subject would immediately begin paced, reciprocal tapping between the two plates. Tapping difficulty was manipulated as in Experiment 1, so that subjects either tapped between wide targets close together or narrow targets relatively far apart.

Five seconds before the end of the retention interval, a $1,000-\mathrm{Hz}$ Sonalert tone was sounded to signal, in the appropriate conditions, that the subject should stop counting or tapping, look at the display screen, and poise his left-hand middle and index fingers above the recognition test response keys which were located to the left of the tapping apparatus. Subjects had been told to indicate whether or not the test word was among the three items presented at the beginning of the trial by pressing the YES or NO key. YES was indicated by the index finger on special piano-type keys which required a movement of $2 \mathrm{~mm}$ and a static force of $55 \mathrm{~g}$ for closure. Six of the 12 trials in each condition were positive trials (the test item was one of the three words shown during the study phase) and six trials were negative (the test item was a new word). Among the six positive trials, each position (top, middle, or bottom, since the words were arranged vertically) was tested twice. The computercontrolled clock permitted latency measurement accuracy within $1 \mathrm{msec}$.

The subjects rested between each 12-trial block while the experimenter read the instructions for the next block. The entire experimental session lasted about $1 \mathrm{~h}$.

\section{Results and Discussion}

The mean reaction times for correct responses on both positive and negative trials for each of the nine conditions are presented in Table 3. The effect of conditions was reliable $[\mathrm{F}(8,136)=12.31, \mathrm{p}<.001, \mathrm{MSe}=$ $200,483]$, as was the effect of positive vs negative trials $[F(1,17)=10.53, p<.01, \mathrm{MSe}=78,439]$. Negative responses were faster than positive responses (mean reaction times of 903 vs $944 \mathrm{msec}$ ), but this may simply have been due to the fact that subjects made negative responses with their left index fingers and positive responses with their left middle fingers. There was no interaction between conditions and positive vs negative response types $[\mathrm{F}(8,36)<1, \mathrm{MSe}=73,159]$.
Of primary interest is the pattern of reaction times in the different conditions. We were assuming that latencies in three conditions, the 0 -sec retention interval condition and the unfilled conditions at the other retention intervals, would provide us with an estimate of retrieval time from primary memory. Latencies following the intervals filled with a backward-counting task, especially the 40-sec interval, were supposed to provide us with an estimate of time to retrieve information from secondary memory. We were assuming, of course, that latencies for retrieval from secondary memory would be greater than from primary memory. The results in Table 3 bear out this assumption; latencies in the backward-counting conditions were about $240 \mathrm{msec}$ longer than combined latencies in the three conditions supposed to involve primary memory.

The critical question is whether latencies after interpolated tapping tasks more closely resemble those after counting backward or those after the unfilled and 0 -sec retention interval conditions or, put differently, whe ther or not information is held in primary or secondary memory after these interpolated tapping tasks. The evidence allows an unequivocal decision. The mean reaction times after 20 or $40 \mathrm{sec}$ of either easy or difficult tapping are much more similar to reaction times after the unfilled and 0-sec retention intervals than after intervals filled with counting backward. Comparisons with Newman-Keuls tests provide statistical justification for these conclusions. The latencies after 20 or $40 \mathrm{sec}$ of counting backward did not differ reliably from one another, but did differ reliably from all other conditions. Furthermore, performance in no other two conditions differed reliably. The conclusion we draw from these results in conjunction with those of the other two experiments is that subjects are able to maintain information in short-term memory (presumably by rehearsal) while simultaneously performing a nonverbal interpolated task.

Presented in Table 4 are the mean reaction times for both correct responses and errors, the number of observations on which each is based, and the standard errors of the mean. The mean correct times are, of course, the same as the mean times in Table 3. The error reaction times are quite unstable since they are based on so few observations, but even here there is a trend for latencies following backward counting to be noticeably

Table 3

Mean Reaction Times on Correct Trials Only for Positive and Negative Responses in the Nine Conditions of Experiment 3

Retention Interval (Seconds)

\begin{tabular}{|c|c|c|c|c|c|c|c|c|c|}
\hline & \multirow[b]{2}{*}{0} & \multicolumn{4}{|c|}{20} & \multicolumn{4}{|c|}{40} \\
\hline & & Unfilled & $\begin{array}{c}\text { Easy } \\
\text { Tapping }\end{array}$ & $\begin{array}{l}\text { Difficult } \\
\text { Tapping }\end{array}$ & $\begin{array}{l}\text { Counting } \\
\text { Backward }\end{array}$ & Unfilled & $\begin{array}{c}\text { Easy } \\
\text { Tapping }\end{array}$ & $\begin{array}{l}\text { Difficult } \\
\text { Tapping }\end{array}$ & $\begin{array}{c}\text { Counting } \\
\text { Backward }\end{array}$ \\
\hline $\begin{array}{l}\text { Positive trials } \\
\text { Negative trials } \\
\text { Mean }\end{array}$ & $\begin{array}{l}906 \\
862 \\
884\end{array}$ & $\begin{array}{l}878 \\
805 \\
841\end{array}$ & $\begin{array}{l}857 \\
876 \\
867\end{array}$ & $\begin{array}{l}892 \\
850 \\
871\end{array}$ & $\begin{array}{l}1169 \\
1107 \\
1138\end{array}$ & $\begin{array}{l}900 \\
865 \\
883\end{array}$ & $\begin{array}{l}877 \\
852 \\
865\end{array}$ & $\begin{array}{l}913 \\
858 \\
885\end{array}$ & $\begin{array}{l}1107 \\
1054 \\
1080\end{array}$ \\
\hline
\end{tabular}


Table 4

Mean Reaction Times for Correct and Incorrect Responses, Standard Errors of the Means, and the Number of Observations on Which Each is Based for the Nine Conditions of Experiment 3

\begin{tabular}{|c|c|c|c|c|c|c|c|c|}
\hline \multirow[b]{2}{*}{ Interpolated Activity } & \multicolumn{3}{|c|}{ Correct } & \multicolumn{3}{|c|}{ Error } & \multicolumn{2}{|c|}{ Percent Errors } \\
\hline & $\mathbf{N}$ & RT & $S_{x}$ & $\mathrm{~N}$ & RT & $S_{x}$ & $\begin{array}{l}\text { Positive } \\
\text { Trials }\end{array}$ & $\begin{array}{c}\text { Negative } \\
\text { Trials }\end{array}$ \\
\hline \multicolumn{9}{|c|}{ 0-Sec Retention Interval } \\
\hline & 213 & 884 & 19.23 & 3 & 570 & 137.15 & 2 & 1 \\
\hline \multicolumn{9}{|c|}{ 20-Sec Retention Interval } \\
\hline Unfilled & 211 & 841 & 19.61 & 5 & 649 & 77.88 & 4 & 1 \\
\hline Easy tapping & 210 & 867 & 18.43 & 6 & 1351 & 245.39 & 5 & 1 \\
\hline Difficult tapping & 208 & 871 & 21.26 & 8 & 1065 & 160.72 & 6 & 2 \\
\hline Counting backward & 198 & 1138 & 33.52 & 18 & 1636 & 143.49 & 16 & 1 \\
\hline \multicolumn{9}{|c|}{ 40-Sec Retention Interval } \\
\hline Unfilled & 211 & 883 & 21.38 & 5 & 1171 & 192.69 & 5 & 0 \\
\hline Easy tapping & 207 & 865 & 18.23 & 9 & 1179 & 170.85 & 7 & 1 \\
\hline Difficult tapping & 206 & 885 & 27.05 & 10 & 1299 & 267.18 & 6 & 3 \\
\hline Counting backward & 204 & 1080 & 29.63 & 12 & 1922 & 164.14 & 11 & 0 \\
\hline
\end{tabular}

Note-Also included are the percentage of errors for positive and negative responses in each condition.

longer than in the other conditions. Also included in Table 4 are the percent errors for positive and negative responses in each of the conditions. There were very few errors in most conditions, the exceptions being for positive responses in the backward-counting conditions. It is not surprising that performance was worse in the backward-counting condition, but the few errors in any condition on negative trials was surprising and suggests a response bias favoring either negative responses or the middle finger.

\section{GENERAL DISCUSSION}

Three assumptions must be validated before one may use interpolated task performance as an index of covert rehearsal, as has been done in studies on the issue of decay in immediate memory. First, the memory task must be shown to demand capacity; second, the interpolated task must be shown to demand capacity; and third, both rehearsal and the interpolated task must be shown to draw on the same limited source of capacity. The present experiments tested these assumptions directly by varying the capacity required by the interpolated task. Since this variation did not affect memory performance, it appears that one or more of the three assumptions is faulty in cases where a nonverbal interpolated task is combined with a verbal memory task. Since verbal memory tasks can easily be shown to require capacity when they are combined with other verbal interpolated tasks (e.g., counting backward), the first assumption does not seem faulty. The second assumption also appears secure, since tapping has been shown to require capacity when combined with other tasks (Kantowitz \& Knight, 1974, 1976a). The faulty assumption is apparently the third. A verbal memory task and a nonverbal interpolated task seem to draw capacity from different sources. We feel safe in general- izing the implications of our results to those experiments where tone detection was the interpolated task. In these experiments not only is the third assumption suspect, but there is even great doubt about whether or not the second assumption holds for detection tasks, as already discussed.

The conclusion to be drawn from our experiments is that a verbal memory task and nonverbal interpolated task can be successfully combined, with no capacityrelated decrement in performance on either task when the difficulty of interpolated task is varied. The slight decrement in recall exhibited when a memory task is combined with a nonverbal interpolated task relative to the memory-only condition can be attributed to qualitative differences in the tasks, since capacity requirements of the interpolated task have no effect on recall.

The present experiments were not designed to elucidate the nature of the qualitative differences in the memory task when performed alone vs in combination with the tapping task, so we can only speculate as to why performance was worse in the dual- than in the single-task conditions in Experiments 1 and 2. However, this finding appears to be quite reliable, not only because of its replication in the present experiments but also since Kantowitz (1972) obtained an identical result with a tapping interpolated task and a motor short-term memory task. There are at least two possible reasons for such qualitative differences. First, subjects may feel that the memory task is more important in single-task conditions than when it is combined with another task. So, perhaps in dual-task conditions subjects were simply less motivated to do well on the memory task, since they evaluated their own performance on both tasks jointly. A second possibility might be slight differences in recall procedure between single- and dual-task conditions. In Experiments 1 and 2, subjects in dual-task conditions tapped with their right 
hands during the retention interval and then, when a recall tone sounded, had to put down the tapping stylus, locate and reach for a pencil, and begin writing their responses. In unfilled conditions subjects could prepare for the recall tone in advance and immediately begin writing when the tone sounded. This possibility is bolstered somewhat by the results of Experiment 3, where a tone sounded $5 \mathrm{sec}$ before the recognition test item appeared in all conditions to alert the subjects for the test. Under these conditions, there was no difference between single- and dual-task performance.

The present results seem to conflict with those reported by Crowder $(1967 \mathrm{a}, \mathrm{b})$, but we think this discrepancy can be readily accounted for by consideration of task differences. In Crowder's (1967a) first two studies, subjects performed a serial keypressing task while attempting to remember five words. The interpolated task required subjects to watch for which of 10 lights came on and then to press one of 10 appropriate keys in order to turn off the light. The difficulty of the interpolated task was manipulated by changing the compatibility of the mappings between the stimulus lights and response keys and by changing the coherence (the repetition pattern) of the stimulus-light sequences. Crowder (1967a) found poorer recall with the lowcompatibility (difficult) than high-compatibility (easy) interpolated task. Also unlike the present results, Crowder (1967a, Table 1) found a tendency for greater dual-task decrements than single-task decrements on the low-compatibility version of the interpolated task. Although the interaction between task condition (singlevs dual-task) and interpolated task difficulty did not attain statistical significance, the pattern of results still appears to contradict our own findings of equal dual-task decrements for easy and difficult versions of the tapping task.

Quite to the contrary, however, we feel that the conflict between the present results and those of Crowder (1967a) provides increased justification to our hesitancy about accepting the general validity of Assumption 3. We think the capacity demands of the interpolated task Crowder (1967a) used were quite different from those used both in the present experiments and in others investigating decay in short-term memory (especially the detection tasks of Reitman and Shiffrin). It may well be that with Crowder's more complex interpolated task it cannot be assumed that the task is strictly nonverbal, especially at low levels of practice (Fleishman \& Hemple, 1954). Under conditions of low stimulus-response compatibility and practice, it is quite likely that subjects used verbal rules to mediate performance ("third light from the left goes with second key from the right," and so forth). Under such conditions both the memory task and serial reaction interpolated task may have utilized the same source of capacity as required by Assumption 3.

Our conclusion that the validity of Assumption 3 is the key to the discrepancy between our results and those of Crowder is supported by two other results. In another study, Crowder (1967b) again combined memory and serial reaction tasks but varied the difficulty of the interpolated reaction task in a different way. Stimulus-response compatibility was always high. In this case, reaction-task performance was worse in dual-task conditions than in single-task conditions, but this dual-task decrement was not greater for the difficult version of the interpolated reaction task than for the easy version. This increases our belief that our present results reflect a real failure of Assumption 3, rather than an inadequate test. Finally, memory task performance in the third experiment (especially reaction time) did decline drastically when a verbal interpolated task was employed. With such an interpolated task, Assumption 3 was probably valid, thus accounting for the observed interference.

The series of studies begun by Reitman (1971) is based on the clever idea of seeing what happens to information over time while a subject's capacity is demanded, but while there is no verbal activity to provide retroactive interference. Unfortunately, the view of central processing capacity which this logic assumes has been found faulty in the present experiments, and if one accepts the more reasonable view that there are various independent components or pools of capacity affecting different stages in performing any task, it may be difficult to apply Reitman's (1971) logic to the issue. If the nature of the interpolated task is entirely different from the verbal recall task, then the experiment is open to attack on the grounds that the two tasks demand different sorts of capacity. Thus, the interpolated task neither affects nor reflects amount of rehearsal. On the other hand, if the two tasks obviously require the same sort of capacity, such as verbal recall and counting backward, a good case can be made that forgetting is due to retroactive interference supplied by the interpolated task. We see no simple way around this impasse and think that, if the issue of decay in shortterm memory is deemed worthy of being further pursued, it may well have to be in a different experimental guise.

\section{REFERENCES}

Brooks, L. R. Spatial and verbal components of the act of recall. Canadian Journal of Psychology, 1968, 22, 349-368.

Brown, J. Some tests of the decay theory of immediate memory. Quarterly Journal of Experimental Psychology, 1958, 10, 12-21.

CrowDer, R. G. Short-term memory for words with a perceptual-motor interpolated activity. Journal of Verbal Learning and Verbal Behavior, 1967, 6, 753-761. (a)

Crowder, R. G. Reciprocity of retention and interpolatedtask scores in short-term memory. Perceptual and Motor Skills, 1967, 24, 903-909. (b)

FitTs, $P$. M. The information capacity of the human movement system in controlling the amplitude of movement. Journal of Experimental Psychology, 1954, 47, 381-391.

Fleishman, E. A., \& Hemple, W. E. Changes in factor 
structure of a complex psychomotor task as a function of practice. Psychometrika. 1954, 19, 239-252.

Johnston, ' $'$. A., Greenberg, S. N., Fisher, R. P., \& Martin, D. W. Divided attention: A vehicle for monitoring memory processes. Journal of Experimental Psychology. 1971, 83, 164-171.

Kahneman, D. Attention and effort. Englewood Cliffs, N.J: Prentice-Hall, 1973.

KalsbeEk, J. W. H., \& SyKes, R. N. Objective measurement of mental load. In A. F. Sanders (Ed.), Attention and performance I. Amsterdam: North-Holland, 1970.

KanTowitz, B. H. Interference in short-term motor memory: Interpolated task difficulty, similarity, or activity? Journal of Experimental Psychology, 1972, 95, 264-274.

KaNTowitz, B. H. Double stimulation. In B. H. Kantowitz (Ed.), Human information processing: Tutorials in performance and cognition. Hillsdale, N.J: Lawrence Eribaum, 1974.

Kantowitz, B. H., \& KNight, J. L. Testing tapping timesharing. Joumal of Experimental Psychology, 1974, 103, 331-336.

Kantowitz, B. H., \& KNIGHT, J. L. Testing tapping timesharing: II. Auditory secondary task. Acta Psychologica, 1976, 40, 343-362. (a)

Kantowitz, B. H., \& KnIGet, J. L. On experimenter-limited processes. Psychological Review, 1976, 83, 502-507. (b)

KePPEL, G.. \& UNDERWOOD, B. J. Proactive inhibition in shortterm retention of single items. Jourial of Verbal Learning and Verbal Behavior, 1962, 1, 153-161.

KERR, B. Processing demands during mental operations. Memory \& Cognition, 1973, 1, 401-412.

McGeoch, J. A. Forgetting and the law of disuse. Psychological Review, 1932, 39, 352-370.
Norman, D. A., \& Bobrow, D. G. On data-limited and resource-limited processes. Cognitive Psychology, 1975, 7, 44-64.

Peterson, L. R., \& Peterson, M. G. Short term retention of individual items. Journal of Experimental Psychology, 1959, 58, 193-198.

PEW, R. W. Human perceptual-motor performance. In B. $H$. Kantowitz (Ed.), Human information processing: Tutorials in performance and cognition. Hillsdale, N.J: Lawrence Erlbaum. 1974.

ReItman, J. S. Mechanisms of forgetting in short term memory. Cognitive Psychology, 1971, 2, 185-195.

Reitman, J. S. Without surreptitious rehearsal, information in short-term memory decays. Journal of Verbal Learning and Verbal Behavior, 1974, 13, 365-377.

ShIFFRIN, R. M. Information persistence in short-term memory. Journal of Experimental Psychology, 1973, 100, 39-49.

Shiffrin, R. M., \& Gardner, G. T. Visual processing capacity and attentional control. Journal of Experimental Psychology, 1972, 93, 72-82.

Sorkin, R. D., \& Pohlmann, L. D. Some models of observer behavior in two-channel auditory signal detection. Perception \& Psychophysics, 1973, 14, 101-109.

Watkins, M. J., Watkins, O. C., Craik, F. I. M., \& MAzURYK, G. Effect of nonverbal distraction on short-term storage. Joumal of Experimental Psychology, 1974, 101, 296-300.

Woodworth, R. S. Psychology. New York: Holt, 1929.

(Received for publication April 16, 1976 revision accepted September 27, 1976.) 